\title{
Design specification representation for intelligent product appearance design
}

\author{
Hu Huicong ${ }^{1 *}$, Lu Wen-Feng ${ }^{2}$ \\ ${ }^{1}$ Department of Design, Harbin Institute of Technology (Shenzhen), Shenzhen, Guangdong, 518000, China \\ ${ }^{2}$ Department of Mechanical Engineering, National University of Singapore, Singapore, Singapore, 117575, Singapore
}

\begin{abstract}
Traditional intelligent product design usually focuses on functional design, aiming to generate appropriate structures that would provide required functions. Design specifications are mainly formulized into technical descriptions or values that are related to certain functional or usability requirements. In today's global market, to in-crease user satisfaction, the appearance design of a product become vital for users to make purchasing decisions. This is particularly true of today's consumer products such as mobile phones, digital cameras, and other electronic products. In intelligent product appearance design, design specifications are merely described as basic geometric dimensions and types of surface materials. Additionally, the aesthetic considerations and emotional needs of product appearance are seldom discussed when establishing design specifications for product appearance design. In this regard, the objective of this study is to propose a design specification representation framework for intelligent product appearance design considering both emotional and aesthetic aspects. The framework be-gins to investigate user needs by acquiring user aesthetic experience. Based on the notion of aesthetic experience, a detailed representation model of appearance design specifications is provided for satisfying user emotional and aesthetic needs. Finally, a case study of the appearance design of digital cameras is provided to demonstrate the acquisition of user needs and establishment of design specifications based on the proposed framework.
\end{abstract}

\section{Introduction}

Traditional intelligent product design usually focuses on functional design, aiming to generate appropriate structures that would provide required functions (A. Chakrabarti \& Bligh, 1996; Umeda \& Ishii, 1996). To interpret user needs into product artifact, design specifications act as the intermediate that transfers "the language of the users" into technical descriptions of product requirements during conceptual design (Ulrich and Eppinger 2012). In most conceptual design studies, design specifications are mainly formulized into technical descriptions or values that are related to certain functional or usability requirements (Deng 2002, Barnum and Mattson 2010, Komoto and Tomiyama 2012). In today's global market, user satisfaction has become one of the major determinants leading to the success of a product (Chen \& Chuang, 2008; G. C. Smith \& Smith, 2012). Functional aspect of a product is no longer the only primary concern for users to make purchasing decisions. To increase user satisfaction, the appearance design of a product should be taken into consideration as well (Barnes \& Lillford, 2009). This is particularly true of today's consumer products such as mobile phones, digital cameras, and other electronic products. However, appearance design specifications are merely described as basic geometric dimensions and types of surface materials. Additionally, the aesthetic considerations and emotional needs of product appearance are seldom discussed when establishing design specifications for product appearance design.

In product appearance design, identifying user needs at the conceptual design stage plays a vital role in raising the overall user satisfaction and avoiding expensive failures. This is due to the fact that user needs are regarded as a basis for establishing design specification, generating product concepts, and criteria for concept evaluation and validation. However, capturing this aspect of user needs is inherently tough. Users usually have ambiguous ideas of how they want the product to be, and use non-technical words to express their ideas and needs. As a result, it is not easy to transfer this aspect of user needs into quantified design specifications for intelligent product appearance design.

A scientific and efficient way of capturing user aesthetic needs and establishing quantified appearance design specifications is to access user responses to the visual appearance of design elements (Chen \& Chuang, 2008; Coates, 2003). This way allows the design team to obtain user needs by investigating user responses, and generate appearance design specifications based on obtained user needs. To investigate users' affective response to a product, Nagamachi (1995) developed Kansei Engineering that translates user psychological feelings into product form elements. Following this approach, many other Kansei Engineering related studies

\footnotetext{
*Corresponding author's e-mail: huhuicong@hit.edu.cn
} 
have been proposed (Barone, Lombardo, \& Tarantino, 2007; Chen \& Chuang, 2008; S. Smith, Smith, \& Chen, 2013). Although these methods have been successfully applied in handling the emotional aspect of user needs, one limitation remains in the studies of Kansei Engineering is that Kansei Engineering lacks the considerations of the aesthetic aspect of user needs and design specifications. In Kansei Engineering, user needs of a product appearance are commonly described by Kansei words, which are usually adjectives, nouns, or verbs that indicate certain human emotions associated with the product form. By constructing the relationships between Kansei words and design elements, Kansei Engineering could be effective in identifying certain design elements that match user preferred Kansei words based on existing product forms. However, it could not support the establishment of design specifications to constitute an aesthetically pleasing form. As a result, the combination of identified design elements may not be aesthetic with the initial arrangement of design elements.

\section{Aesthetic Experience}

Aesthetic experience is a complicated concept that encompasses all processes involved in the interaction between user and visual parts of a product. Leder, Belke, Oeberst, and Augustin (2004) proposed aesthetic experience as a series of activities that contain perceptual analysis, comparison with previous encounters, classification, interpretation, and evaluation, and finally resulting in aesthetic judgments and emotions. During the interaction, the expression of design information and the inherent arrangement of visual design elements serve as two main indicators influencing user aesthetic experience (Schifferstein \& Hekkert, 2008). Similar conclusions can be found in opinions of Lavie and Tractinsky (2004)'s "classical aesthetics and expressive aesthetics", Coates (2003)'s "information and concinnity", and Crilly et al. (2004)'s "aesthetic impression, semantic interpretation and symbolic association of user cognitive response".

The expression of design information impacts the way that people understands and interprets product forms (Schifferstein \& Hekkert, 2008). This indicator focuses on properties of intangible meanings and metaphors contained in product forms. The user perception of this indicator is very subjective and depends highly on users' personal background and life experience, including one's age, gender, personality, social status, culture, etc. (Crilly et al., 2004; Holbrook \& Schindler, 1994). Additionally, the typicality and novelty of the product form and the use of certain design metaphors or expressions also play a role in affecting the user perception of the product (Noble \& Kumar, 2010; Schifferstein \& Hekkert, 2008).

The inherent arrangement of visual design elements (i.e., form, color, texture, etc.) determines the universal attractiveness of a product. Certain lines, proportions and color combinations will be regarded as "aesthetic" according to human cognition (Ralls, 1995). This indicator reflects the notion of beauty perceived by human sensors and is considered as an objective property in affecting the aesthetic experience. Many design theories related to this indicator has been introduced such as the golden section (Elam, 2001). One famous pioneer of product design is the Bauhaus' teaching theory which is embraced Gestalt psychology and attempt to build a new sensitivity based on design elements such as line, color, text, etc. (Kim, 2006). In Gestalt psychology, scholars believed that "the perception of the whole is greater than a sum of individual parts" (Katz, 1950). In another word, people turn to perceive things that are orderly, balanced and unified, providing an overall sense of feeling (D. Chang \& Nesbitt, 2006). However, a very simple and orderly design will be regarded as boredom and tedious (Schifferstein \& Hekkert, 2008). Sometimes, complexity and variety are required to generate arousal in user perception (Berlyne, 1966). An aesthetic balance is suggested to be lies somewhere between concinnity order and complex arousal (Gombrich, 1984). To drive in both arousal-reducing and arousaldriving direction of design constitution, designers usually refer to aesthetic principles for the arrangement of design elements. Aesthetic design principles are universal acknowledged design strategies for visual appearance design. They can help designers to effectively arrange visual design elements to reflect order and complex (Wallschlaeger et al., 1992). Stebbing (2004) summarized the basic aesthetic design principles on visual composition as contrast, rhythm, balance, and proportion (CRBP). Other frequently-used principles may share some similar purposes with CRBP and could be grouped under them.

\section{Framework}

In this section, a framework is proposed to represent design specification including both emotion and aesthetic aspects. Figure 1 illustrates the proposed framework. For a three-dimensional product appearance design, design specifications are described regarding each view (e.g. front, back, top, bottom, left, and right view) of the product appearance. In a single view, design specifications are classified into two categories, i.e. emotion and aesthetic indicator.

Emotion describes design specifications of expressing subjective emotions and meanings in the product appearance. Emotional words are used to evaluate the expression of emotions. The words can be either adjectives to describe certain emotional meanings or nouns to indicate metaphors if certain objects. According to Dong et al. (2001), four types of adjective words are selected to group emotional words, namely are categories of physiological, psychological, cultural, and physical. Based on the emotional object categories, three types (abstract, inanimate, and animate) are used to indicate design metaphors. To quantify emotion design specifications, the value of each emotion attribute is defined as the degree that people agree on the meaning of the emotion word from the product appearance. For example, the emotion attribute "modern" can be defined to have values of "very high=5", "high=4", "middle=3", "low $=2$ ", and "very low $=1$ ". If the value of the emotion 
attribute "modern" is "very low $=1$ ", it indicates that the product does not look "modern" to people at all.

Aesthetic indicator summarizes the aesthetic strategies to be adopted for design constitution. Aesthetic design principles are used as criteria to quantify design specifications of arranging design elements. Each aesthetic design principle is regarded as the attribute of

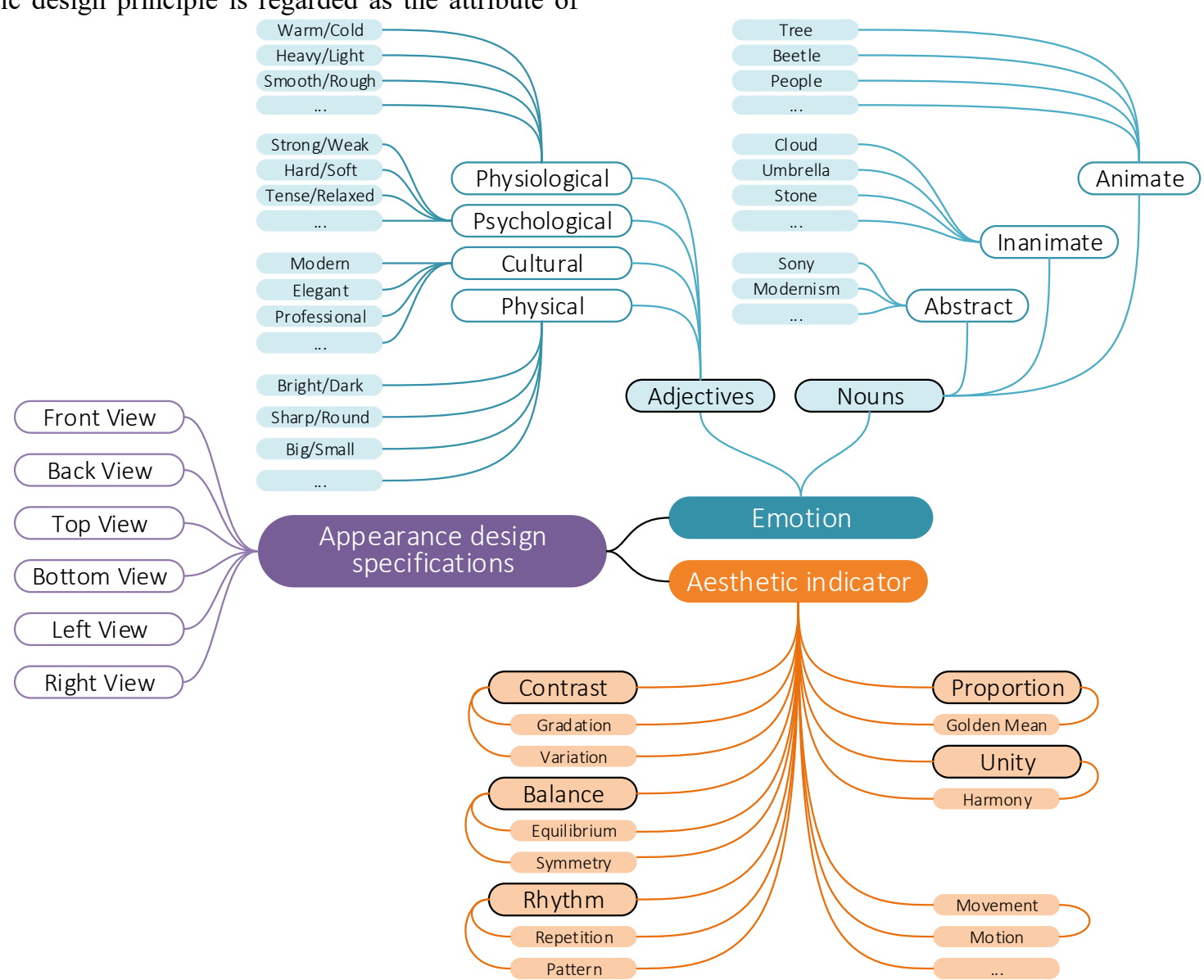

Figure 1. Proposed framework of design specifications representation in product appearance design.

\section{Case Study}

A case study was conducted to illustrate the proposed framework for the acquisition of user needs and establishment of design specifications based on aesthetic experiences of users and designer(s). In the case study, eight interchangeable-lens digital cameras were selected as design samples. The design samples were selected with different styling forms, colors, and texture combinations. aesthetic. Frequently used aesthetic design principles (contrast, balance, rhythm, proportion, and unity) are listed in the representation framework based on Stebbing (2004)'s study. The value of each aesthetic attribute is defined as the degree of implementing the corresponding aesthetic design principle in the product appearance.
Emotion adjectives "classic", "modern", "elegant", "cute", and "professional" were chosen as emotion attributes and aesthetic design principles "contrast" and "balance" were selected as aesthetic attributes of design specifications (Figure 2). Fuzzy sets of Fuzzy Logic which is a modeling tool and well suited for modeling imprecise information (Patyra \& Mlynek, 1996) were used to quantify the values of design specifications. Triangular form of the membership function was employed to determine the linguistic criteria of fuzzy sets (Table 1).

Table 1. Linguistic criteria for appearance specification attributes.

\begin{tabular}{|l|l|}
\hline Linguistic value & Interval of triangular fuzzy number \\
\hline Very low (VL) & {$[0,0.167]$} \\
\hline Low (L) & {$[0,0.333]$} \\
\hline Medium low (ML) & {$[0.167,0.5]$} \\
\hline Medium (M) & {$[0.333,0.667]$} \\
\hline Medium high (MH) & {$[0.5,0.833]$} \\
\hline High (H) & {$[0.667,1]$} \\
\hline Very high (VH) & {$[0.833,1]$} \\
\hline
\end{tabular}

To acquire the value of emotion attributes, semantic differential (SD) method, which is a self-report method us- ing a Likert scale, was employed to evaluate design samples regarding each emotion attribute. A seven-point 
SD scale (Low, Media Low to 7) was employed in the user experiment to acquire user aesthetic experience. Fortythree female college students were selected to participate the user experiment. The user evaluation was done by 43 female that from target users. The evaluation results are shown in Table 2.

Table 2. Values of emotion attributes.

\begin{tabular}{|l|l|l|l|l|l|}
\hline Design sample & Classic & Modern & Elegant & Cute & Professional \\
\hline 1 & L & H & H & MH & M \\
\hline 2 & H & ML & M & L & MH \\
\hline 3 & L & H & ML & H & ML \\
\hline 4 & ML & MH & ML & M & ML \\
\hline 5 & MH & ML & MH & L & H \\
\hline 6 & M & ML & ML & ML & M \\
\hline 7 & ML & MH & M & ML & MH \\
\hline 8 & ML & MH & H & M & ML \\
\hline
\end{tabular}

To obtain the value of aesthetic attributes, Analytic Hierarchy Process (AHP) developed by Saaty (1988) was applied to perform comparative judgment on the degree of implementing aesthetic design principles in design samples. Pairwise comparisons were performed between design samples regarding each aesthetic design principle based on the intensity of importance scale shown in Table 3 . The local weight on the degree of implementing each aesthetic design principle was computed. This process was done by one product designer with more than five years' experience on implementing aesthetic design principles. The resulted Linguistic values with degree of support (DOS) of aesthetic attributes are presented in Table 4.

The fuzzy sets obtained from the user experiment and AHP can then be used to construct a fuzzy model for the reasoning process in intelligent product appearance design.

Table 3. Intensity of importance scale for AHP evaluation.

\begin{tabular}{|l|l|l|}
\hline $\begin{array}{l}\text { Intensity of } \\
\text { importance }\end{array}$ & Definition & Explanation \\
\hline 1 & Equal importance & Two activities contribute equally to the objective \\
\hline 3 & $\begin{array}{l}\text { Weak importance of one over } \\
\text { another }\end{array}$ & $\begin{array}{l}\text { Experience and judgment slightly favor one activity over } \\
\text { another }\end{array}$ \\
\hline 5 & Essential or strong importance & $\begin{array}{l}\text { Experience and judgment strongly favor one activity } \\
\text { over another }\end{array}$ \\
\hline 7 & Demonstrated importance & $\begin{array}{l}\text { An activity is strongly favored and its dominance } \\
\text { demonstrated in practice }\end{array}$ \\
\hline 9 & Absolute importance & $\begin{array}{l}\text { The evidence favoring one activity over another is of the } \\
\text { highest possible order of affirmation }\end{array}$ \\
\hline $2,4,6,8$ & $\begin{array}{l}\text { Intermediate values between the two } \\
\text { adjacent judgments }\end{array}$ & When compromise is needed \\
\hline
\end{tabular}

Table 4. AHP results of the fuzzy set of aesthetic indicator attributes

\begin{tabular}{|c|c|c|c|c|}
\hline \multirow{2}{*}{ Design sample } & \multicolumn{2}{|l|}{ Contrast } & \multicolumn{2}{|c|}{ Balance } \\
\hline & \multicolumn{2}{|c|}{ Linguistic value $\backslash \mathrm{DOS}$} & \multicolumn{2}{|c|}{ Linguistic value $\backslash \mathrm{DOS}$} \\
\hline 1 & $\mathrm{~L} \backslash 0.70$ & VL\} \backslash 0 . 3 0 $&{\mathrm{MH} \backslash 0.76} &{\mathrm{H} \backslash 0.24} \\
{\hline 2} &{\text { VL\0.55 }} &{\mathrm{L} \backslash 0.45} &{\mathrm{~L} \backslash 0.98} &{\text { VL\} \backslash 0 . 0 2 } \\
{\hline 3} &{\mathrm{H} \backslash 0.50} &{\text { VH } \backslash 0.50} &{\mathrm{MH} \backslash 0.61} &{\mathrm{M} \backslash 0.39} \\
{\hline 4} &{\mathrm{MH} \backslash 0.90} &{\mathrm{H} \backslash 0.10} &{\text { ML\0.86 }} &{\mathrm{M} \backslash 0.14} \\
{\hline 5} &{\text { ML } \backslash 0.54} &{\mathrm{~L} \backslash 0.46} &{\mathrm{H} \backslash 1} &{\mathrm{VH} \backslash 0} \\
{\hline 6} &{\mathrm{M} \backslash 0.92} &{\text { ML\0.08 }} &{\mathrm{L} \backslash 0.61} &{\text { ML\0.39 }} \\
{\hline 7} &{\text { VL } \backslash 0.58} &{\mathrm{~L} \backslash 0.42} &{\text { ML\0.81 }} &{\mathrm{L} \backslash 0.19} \\
{\hline 8} &{\mathrm{~L} \backslash 0.62} &{\text { VL\} \backslash 0 . 3 8 } &{\mathrm{L} \backslash 0.82} &{\text { ML\0.18 }} \\
{\hline} &{\text { Inconsistency }=0.04} &{ } &{\text { Inconsistency }=0.07} &{ } \\
\hline\end{array}$
\end{tabular}

\section{Conclusion}

The aesthetic aspect of product design becomes important in increasing user satisfaction. Information repre-sentation of design specifications in product appearance design is seldom discussed in the literature. To address this issue, a representation frame of appearance design specifications was proposed based on the notion of aes-thetic experience. A digital camera design case study was conducted to illustrate the proposed framework. The case study 
reflected that the framework is effective in quantifying emotion and aesthetic design information of appearance design specifications. In the future, a fuzzy logic model between design specifications and user prefer-ences can be constructed to address the reasoning process for intelligent product appearance design.

\section{References}

1. Barnes, C. \& Lillford, S.P., 2009. Decision support for the design of affective products. Journal of Engineering Design, 20 (5), 477-492.

2. Barnum, G.J. \& Mattson, C.A., 2010. A computationally assisted methodology for preference-guided conceptual design. Journal of Mechanical Design, 132 (12), 121003.

3. Barone, S., Lombardo, A. \& Tarantino, P., 2007. A weighted logistic regression for conjoint analysis and kansei engineering. Quality and Reliability Engineering International, 23 (6), 689-706.

4. Berlyne, D.E., 1966. Curiosity and exploration. Science, 153 (3731), 25-33.

5. Chakrabarti, A. \& Bligh, T.P., 1996. An approach to functional synthesis of mechanical design concepts: Theory, applications, and emerging research issues. AI EDAM, 10 (4), 313-331.

6. Chang, D., \& Nesbitt, K. V. (2006, October). Identifying commonly-used gestalt principles as a design framework for multi-sensory displays. In 2006 IEEE International Conference on Systems, Man and Cy-bernetics (Vol. 3, pp. 2452-2457). IEEE..

7. Chen, C.-C. \& Chuang, M.-C., 2008. Integrating the kano model into a robust design approach to enhance customer satisfaction with product design. International Journal of Production Economics, 114 (2), 667-681.

8. Coates, D., 2003. Watches tell more than time: Product design, information, and the quest for elegance New York: McGraw-Hill.

9. Crilly, N., Moultrie, J. \& Clarkson, P.J., 2004. Seeing things: Consumer response to the visual domain in product design. Design Studies, 25 (6), 547-577 Available from: http://www.sciencedirect.com/science/article/pii/S0 $142694 X 04000225$.

10. Deng, Y.M., 2002. Function and behavior representation in conceptual mechanical design. AI EDAM, 16 (5), 343-362.

11. Elam, K., 2001. Geometry of design: Studies in proportion and composition New York: Princeton Archi-tectural Press.

12. Gombrich, E.H., 1984. The sense of order: A study in the psychology of decorative art Oxford: Phaidon.

13. Hekkert, P., 2006. Design aesthetics: Principles of pleasure in design. Psychology Science, 48 (2), 157172.

14. Holbrook, M.B. \& Schindler, R.M., 1994. Age, sex, and attitude toward the past as predictors of con- sumers' aesthetic tastes for cultural products. Journal of Marketing Research, 31 (3), 412-422.

15. Katz, D., 1950. Gestalt psychology, its nature and significance New York: Ronald Press Co.

16. Kim, N., 2006. A history of design theory in art education. Journal of Aesthetic Education, 40 (2), 12-28.

17. Komoto, H. \& Tomiyama, T., 2012. A framework for computer-aided conceptual design and its application to system architecting of mechatronics products. Computer-Aided Design, 44 (10), 931-946 Available from:

http://www.sciencedirect.com/science/article/pii/S0 010448512000401 .

18. Lavie, T. \& Tractinsky, N., 2004. Assessing dimensions of perceived visual aesthetics of web sites. In-ternational Journal of Human - Computer Studies, 60 (3), 269-298.

19. Leder, H., Belke, B., Oeberst, A. \& Augustin, D., 2004. A model of aesthetic appreciation and aesthetic judgments. British journal of psychology (London, England : 1953), 95 (Pt 4), 489.

20. Nagamachi, M., 1995. Kansei engineering: A new ergonomic consumer-oriented technology for product development. International Journal of Industrial Ergonomics, 15 (1), 3-11.

21. Noble, C.H. \& Kumar, M., 2010. Exploring the appeal of product design: A grounded, value-based model of key design elements and relationships: Exploring the appeal of product design. Journal of Product Innovation Management, 27 (5), 640-657.

22. Patyra, M.J. \& Mlynek, D.M., 1996. Fuzzy logic: Implementation and applications Wiesbaden: Vieweg+Teubner Verlag.

23. Ralls, K., 1995. But is it science? Conservation Biology, 9 (5), 983-984.

24. Saaty, T.L., 1988. What is the analytic hierarchy process? In Gautam, M. ed. Mathematical models for decision support. Springer-Verlag New York, Inc., 109-121.

25. Schifferstein, H.N.J. \& Hekkert, P., 2008. Product experience San Diego, Ca: Elsevier.

26. Smith, G.C. \& Smith, S., 2012. Latent semantic engineering - a new conceptual user-centered design approach. Advanced Engineering Informatics, 26 (2), 456-473.

27. Smith, S., Smith, G.C. \& Chen, Y.-R., 2013. A kelsa approach for user-centered design. Journal of Intelligent Manufacturing, 24 (5), 919-933.

28. Stebbing, P.D., 2004. A universal grammar for visual composition? Leonardo, 37 (1), 63-70 Available from:

http://www.jstor.org.libproxy1.nus.edu.sg/stable/15 77576.

29. Ulrich, K.T. \& Eppinger, S.D., 2012. Product design and development New York, NY: McGrawHill/Irwin.

30. Umeda, Y., Ishii, M., Yoshioka, M., Shimomura, Y. \& Tomiyama, T., 1996. Supporting conceptual design based on the function-behavior-state modeler. AI EDAM, 10 (4), 275-288. 
31. Wallschlaeger, C., Busic-Snyder, C., \& Morgan, M. (1992). Basic visual concepts and principles for artists, architects, and designers. Wm. C. Brown Publishers. 